\title{
Tumor-suppressive role of HACE1 in hepatocellular carcinoma and its clinical significance
}

\author{
ZENG-FA GAO ${ }^{1,5}$, YONG-NA WU ${ }^{2}$, ZHONG-TIAN BAI ${ }^{3}$, LEI ZHANG $^{3}$, QI ZHOU $^{4}$ and XUN $\mathrm{LI}^{2,3}$ \\ ${ }^{1}$ The First Clinical Medical College, Lanzhou University; ${ }^{2}$ Gansu Province Key Laboratory of Biotherapy \\ and Regenerative Medicine; ${ }^{3}$ The Second Department of General Surgery, The First Hospital of Lanzhou University; \\ ${ }^{4}$ Department of Pathology, The First Hospital of Lanzhou University (The Branch Hospital of Donggang), Lanzhou, \\ Gansu 730000; ${ }^{5}$ The Second Department of General Surgery, Gaomi People's Hospital, Weifang, Shandong 261000, P.R. China
}

Received March 17, 2016; Accepted April 13, 2016

DOI: $10.3892 /$ or.2016.5205

\begin{abstract}
An increasing body of evidence suggests that downregulation or deletion of HECT domain and ankyrin repeat containing E3 ubiquitin protein ligase 1 (HACE1) gene plays an important role in the occurrence, invasion and metastasis process in many human malignancies and is closely related to prognosis. However, sparse evidence exists concerning the precise function and clinical significance of HACE1 in hepatocellular carcinoma (HCC). In the present study, we investigated the expression pattern of HACE1 in HCC tissues and cell lines, and determined the potential functions of HACE1 in HCC cell lines and evaluated the relationships between HACE1 expression and clinicopathological characteristics. Protein and mRNA expression levels of HACE1 in human HCC tissues and cell lines were examined by western blot analysis, quantitative real-time polymerase chain reaction and immunohistochemical (IHC) analyses. IHC was used to analyze the correlations between HACE1 expression and clinicopathological features. HACE1 was upregulated in SMCC7721 cells by transfection with pcDNA3.1-HACE1 and Huh7 cells were transfected with siRNA targeting HACE1 for downregulation. Cell Counting Kit-8, Transwell and wound healing assays were performed to investigate the effects of the overexpression and knockdown of HACE1 on cellular proliferation and migration. The results revealed that HACE1 expression was lower in the HCC tissues and cell lines at the mRNA and protein levels compared to levels noted in the matched non-tumor tissues and the normal liver cell line L02. Knockdown of HACE1 in Huh7 cells accelerated cell proliferation and migration $(\mathrm{P}<0.05)$, and overexpression of HACE1 in SMCC7721 cells was found to
\end{abstract}

Correspondence to: Dr Xun Li, The Second Department of General Surgery, The First Hospital of Lanzhou University, 1 Donggang West Road, Lanzhou, Gansu 730000, P.R. China E-mail: 1xdr21@126.com

Key words: HECT domain and ankyrin repeat containing E3 ubiquitin protein ligase 1, hepatocellular carcinoma, tumor suppressor, clinicopathologic characteristics, prognosis decrease the capacity for proliferation and migration $(\mathrm{P}<0.01)$. The results of IHC suggested that the HACE1 expression level was closely related to the serum AFP level, tumor differentiation and vascular invasion $(\mathrm{P}<0.05)$. Patients with low HACE1 expression levels exhibited poorer overall survival and HACE1 was found to be an independent prognostic factor for survival. In conclusion, as a tumor suppressor, HACE1 may be a valuable prognostic biomarker and potential therapeutic target for HCC treatment.

\section{Introduction}

Hepatocellular carcinoma (HCC) is one of the most common malignant tumors worldwide, and is a serious threat to human life. $\mathrm{HCC}$ has the second highest cancer mortality rate, next to lung cancer. Approximately 782,500 new liver cancer cases and 745,500 deaths occured worldwide in 2012, and most primary liver cancers occurring worldwide are HCC. China is a country with a high incidence of HCC, accounting for about $50 \%$ of the total number of cancer cases and deaths (1). Surgical and liver transplantation are still the best treatment option for suitable patients (2-4). However, most HCC cases are diagnosed at the advanced or unresectable stage. Given the poor prognosis of $\mathrm{HCC}$, new therapeutic strategies and novel therapeutic targets are needed, respectively. But current research on the main pathogenic genes and molecular mechanisms of HCC remain unclear. In addition, coupled with the existence of an ageing population and pollution of the environment, we are faced with the increasingly serious situation of HCC. Therefore, the research for pathogenic genes and molecular mechanisms of HCC play a key role in the prevention and treatment of HCC.

HECT domain and ankyrin repeat containing E3 ubiquitin protein ligase 1 (HACE1) is located on chromosome 6q21 which is one site of frequent mutation of malignant tumors (5-8). HACE1 is an important tumor-suppressor gene, which plays an important role in tumor inhibition by mediating cell autophagy, Rac1 ubiquitination and other mechanisms. It is also involved in a variety of biological functions including heart protection, anti-oxidative stress and cellular dynamics (9-13). HACE1 gene was firstly discovered as closely related to the occurrence of Wilms' tumor (5). Futhermore, more evidence demonstrated that lower expression or mutations of HACE1 are associated 
with a variety of human malignant tumors, including breast cancer, colorectal cancer and lymphomas (14-16). However, there have been few available data concerning the precise function of the HACE1 gene in HCC.

In the present study, we investigated the expression of HACE1 in human HCC tissues and cell lines compared with matched adjacted non-tumor HCC tissues and the normal liver cell line L02. Immunohistochemistry (IHC) was performed to analyze the correlation of HACE1 expression with the clinicopathological features of patients. In addition, Cell Counting Kit-8 (CCK-8), Transwell and wound healing assays were applied to ascertain the role of HACE1 in the proliferation and migration of the HCC cell lines SMCC7721 and Huh7.

\section{Materials and methods}

Patient tissue samples. A total of 40 paired tumor and their adjacted non-tumor tissues were collected from HCC patients who underwent routine surgical resection at The First Hospital of Lanzhou University from 2007 to 2010. The fresh tissues were instantly frozen in liquid nitrogen and subsequently stored at $-80^{\circ} \mathrm{C}$ until RNA and protein extraction for reverse-transcription quantitative polymerase chain reaction. In order to determine the relationships between HACE1 and the clinicopathological characteristics of HCC, an additional 102 paraffin-embedded archived specimens from patients who had undergone surgical resection were incorporated into the study for IHC. These patients included 82 males and 20 females, aged between 30 and 78 years, and the median patient age was 49 years. Histology of the tumor tissues was evaluated by two independent pathologists who were blinded to our examination. The clinicopathological information of all patients were collected for analysis, including age, gender, serum AFP, ALT level, tumor size, tumor number, vascular invasion, HBsAg, tumor differentiation, Child-Pugh and liver cirrhosis. The present study was approved by the Ethics Committee of The First Hospital of Lanzhou University prior to the initiation of the study.

Cell lines and culture. The human HCC cell lines Huh7, MHCC97H, HepG2, MHCC97L, HCCLM3, SMCC7721 and liver cell line L02 were obtained from the Cell Bank of the Chinese Academy of Sciences (Shanghai, China). All cell lines were cultured in Dulbecco's modified Eagle's medium (DMEM), supplemented with $10 \%$ fetal bovine serum (FBS) and $100 \mathrm{U} / \mathrm{ml}$ penicillin and $100 \mathrm{mg} / \mathrm{ml}$ streptomycin (all from Gibco, Shanghai, China). The cells were grown at $37^{\circ} \mathrm{C}$ in a humidified atmosphere of $5 \% \mathrm{CO}_{2}$. The medium was changed every 2-3 days. Before further experiments, the protein and mRNA expression levels of HACE1 in the human HCC cell lines were examined by western blot analysis and quantitative real-time polymerase chain reaction (qRT-PCR), respectively. According to the experimental results, we chose the SMCC7721 and Huh7 cell lines for subsequent experiments.

Cell infection. The Huh7 cells were cultured to 50-60\% confluency in 6-well plates and were transiently transfected with siRNA of HACE1 or negative control (NC) siRNA (GeneChem, Shanghai, China) using Lipofectamine 2000 (Invitrogen, Carlsbad, CA, USA) in accordance with the manufacturer's instructions for HACE1 knockdown. HACE1 siRNAs were: GGGCUACAAUGGGAAUAAAdTdT (si-HACE1-1), GCCCGAGGAUAAUGAAACUdTdT (si-HACE1-2), GCCA GUACCUAAAGAUUCUdTdT (si-HACE1-3). The SMCC7721 cells were transfected with pcDNA3.1-HACE1 or mock plasmid (GeneChem) using Lipofectamine 2000 transfection reagent according to the manufacturer's instructions for HACE1 overexpression. The transfection efficiency of both Huh7 and SMCC7721 cells was evaluated using qRT-PCR and western blot analysis. After $36 \mathrm{~h}$ of incubation, the cells were harvested for further analysis. All transfections were performed in triplicates.

$q R T-P C R$. Total RNA from tissues and all 7 cell lines were extracted using TRIzol reagent (Takara, Dalian, China), according to the manufacturer's instructions. The RNA concentration was detected by NanoDrop 1000 (Thermo Fisher Scientific, Wilmington, DE, USA) and complementary DNA was synthesized utilizing a PrimeScript RT reagent kit (Takara) using $2 \mu \mathrm{g}$ RNA. SYBR-Green dye (Takara) and Corbet Rotor-Gene 3000 thermocycler were used to perform the qRT-PCR reaction, according to the supplied protocol. The primers used were as follows: HACE1 forward, 5'-TCCTTGA ATGTCCTGAGTTGA-3' and reverse, 5'-AATCTGGCTGTC CTGAATGC-3'; and GAPDH forward, 5'-GGCATCCTGGGC TACACTGA-3' and reverse, 5'-GTGGTCGTTGAGGGCA ATG-3'. The amplification conditions of qRT-PCR were set as follows: $95^{\circ} \mathrm{C}$ for $30 \mathrm{sec}, 95^{\circ} \mathrm{C}$ for $5 \mathrm{sec}, 60^{\circ} \mathrm{C}$ for $34 \mathrm{sec}, 95^{\circ} \mathrm{C}$ for $15 \mathrm{sec}$ and a total of 40 cycles. The experiments were repeated in triplicate. Relative expression levels of HACE1 were normalized to GAPDH expression in each sample, and the data were analyzed according to the comparative threshold cycle $\left(2^{-\Delta \Delta \mathrm{Ct}}\right)$ method.

Protein extraction and western blot analysis. Total protein of human HCC tissues and cells were extracted using ice-cold lysis buffer (50 mM Tris, pH 7.4, $150 \mathrm{mM} \mathrm{NaCl,} 1 \%$ SDS, $1 \mathrm{mM}$ EDTA, $1 \%$ NP-40) containing $1 \mathrm{mM}$ protein inhibitor and $1 \mathrm{mM}$ PMSF, for $30 \mathrm{~min}$ on ice. The lysates were centrifuged at $12,000 \mathrm{rpm}$ at $4^{\circ} \mathrm{C}$ for $15 \mathrm{~min}$ and the supernatants were collected. Protein concentration was determinated using the BCA protein assay (Beyotime, Shanghai, China). Protein samples $(50 \mu \mathrm{g} /$ lane $)$ were separated by electrophoresis on $10 \%$ sodium dodecyl sulphate-polyacrylamide gel electrophoresis (SDS-PAGE) and then transferred onto polyvinylidene difluoride (PVDF) membrane filters (Millipore Corp., Billerica, MA, USA) in a wet transfer system (Bio-Rad, Berkeley, CA, USA). PVDF membranes were blocked with $2 \%$ BSA for $1 \mathrm{~h}$ at room temperature. Membranes were incubated with anti-human HACE1 antibody (1:2,000 dilution; Abcam, Cambridge, UK) overnight at $4^{\circ} \mathrm{C}$. The membranes were then incubated with the corresponding secondary antibody $(1: 5,000)$ for $1 \mathrm{~h}$ at room temperature. The immunoreactive bands were visualized using enhanced chemiluminescence (ECL) reagent (Thermo Fisher Scientific, Israel), and the relative protein expression of the membranes was then normalized to the $\beta$-actin levels.

Cell proliferation assay. The CCK- 8 assay (Dojindo Laboratories, Kunamoto, Japan) was used to reflect the proliferation of the cells. For the CCK- 8 assay, $~ 5,000$ viable 
cells were placed into 96-well plates at a final volume of $100 \mu 1$ for each well. Every 24 h, $10 \mu 1$ of CCK-8 solution was added to each well, and the plate was further incubated for $2 \mathrm{~h}$ at $37^{\circ} \mathrm{C}$. The absorbance at $450 \mathrm{~nm}$ was quantitated with a microplate reader. The experiment was performed in 6 replicates.

Cell migration assay. Transwell and wound healing assays were used to evaluate the cell migration capability. The Transwell and migration assays were performed using a 24-well plate, and a Transwell chamber with a polycarbonate filter membrane (Corning Inc., Corning, NY, USA) was placed in the 24-well plate, and the pore size of the filter membrane size was $8 \mu \mathrm{m}$. Firstly, digested cells $\left(5 \times 10^{3} /\right.$ chamber) with $200 \mu 1$ serum-free media were added to the upper compartment, and $400 \mu 1$ DMEM containing 10\% FBS was added to the lower compartment, and further incubated for $24 \mathrm{~h}$ at $37^{\circ} \mathrm{C}$. After incubation, the cells in the upper chamber migrated to the lower surface of the membrane. The cells on the upper membrane were removed carefully with a cotton tip and the penetrated polycarbonic membrane was fixed with $95 \%$ methanol and stained with $0.1 \%$ crystal violet. The number of migrated cells was counted in 6 randomly selected fields under an inverted microscope. Independent experiments were performed in triplicate. For the wound healing assay, tumor cells were seeded in a 6-well plate at a density and incubated to $70-80 \%$ confluency as a monolayer. A cell-free straight line was scratched in the center of the well with a sterile $200-\mu 1$ pipette tip. Similarly, a second straight line was created perpendicular to the first line to produce a cross-shaped cellular gap in each well. The cells were subsequently washed twice with phosphate-buffered saline (PBS) and refreshed with medium containing 5\% FBS. The cells were grown for an additional $72 \mathrm{~h}$. Digital images of the cell gap were captured at different time points using a microscope. The gap distance was quantitatively assessed using software.

Immunohistochemistry. The relationships between HACE1 expression and clinicopathological characteristics of the HCC patients was analyzed using $4-\mu \mathrm{m}$ paraffin-embedded specimens by IHC. After incubation at $60^{\circ} \mathrm{C}$ for $30 \mathrm{~min}$, all tissue sections were deparaffinized in xylene, and then rehydrated by graded ethanol solutions before sodium citrate buffer ( $\mathrm{pH}$ 6.0) was used as an antigen retrieval solution. Endogenous peroxidase activity was blocked with $0.3 \%$ hydrogen peroxide in a humidified chamber for $15 \mathrm{~min}$. After washing for three times, the specimens were incubated with rabbit monoclonal antibody against human HACE1 (1:200, ab18056; Abcam) at $4^{\circ} \mathrm{C}$ overnight. Following washing with PBS for three times, the tissue sections were incubated with a horseradish peroxidase-labeled secondary antibody for $1 \mathrm{~h}$ at room temperature. The specimens were incubated with 3,3'-diaminobenzidine (DAB) solution for $3 \mathrm{~min}$. Finally, the sections were counterstained with hematoxylin. The primary antibody was replaced by PBS as a NC. Tissue specimens were assessed separately by two pathologists who were blinded to any information about the patient background or clinical status. The percentage of positive staining was scored as follows: 1 point $(0-25 \%$, weakly stained); 2 points, $(>25-50 \%$, moderately stained); and 3 points $(>50 \%$, strongly stained). The HACE1 immunostaining score
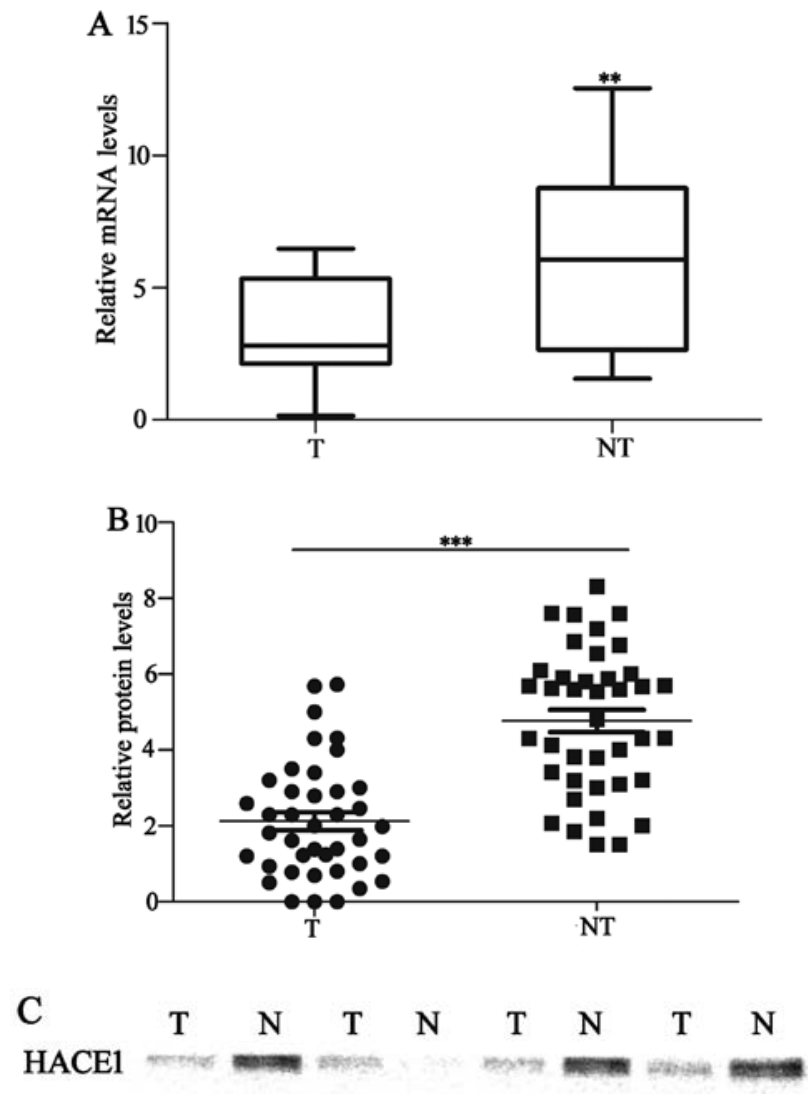

\section{$\beta$-actin}

Figure 1. HACE1 expression in HCC tissues and paired adjacent non-tumor tissues at both the mRNA and protein levels. (A) qRT-PCR analysis of HACE1 mRNA expression in 40 pairs of tumor (T) tissues and the adjacent non-tumor (NT) tissues. (B) HACE1 protein expression levels in HCC tumor specimens and the adjacent non-tumor tissues by western blot analysis. (C) Western blot analysis of the relative HACE1 expression in 4 paired HCC tumor and matched adjacent non-tumor tissues. Expression levels of HACE1 mRNA and protein were significantly decreased in the tumor tissues compared with that observed in the paired adjacent non-tumor tissues $\left({ }^{* *} \mathrm{P}<0.01,{ }^{* * * *} \mathrm{P}<0.001\right)$. HACE1, HECT domain and ankyrin repeat containing E3 ubiquitin protein ligase 1; HCC, hepatocellular carcinoma.

was calculated from the product of the percent positivity score $x$ the staining intensity score which ranged from 0 to 9 . Low HACE1 expression level was defined as a total score of $<5$, and high HACE1 expression was defined as a total score of $\geq 5$.

Statistical analysis. All statistical analysis was performed using SPSS 21.0 software. The relationships between HACE1 expression and clinicopathological characteristics were analyzed using the $\chi^{2}$ test. Student's t-test was used to compare the statistical significance in the various groups. Survival curves were calculated using the Kaplan-Meier method and the result was compared using the log-rank test. Cox's proportional hazard analysis was used to explore univariate and multivariate survival. A difference was considered statistically significant at $\mathrm{P}<0.05$.

\section{Results}

Expression of HACE1 protein and mRNA in HCC and paired adjacent non-tumor tissues. The expression of 

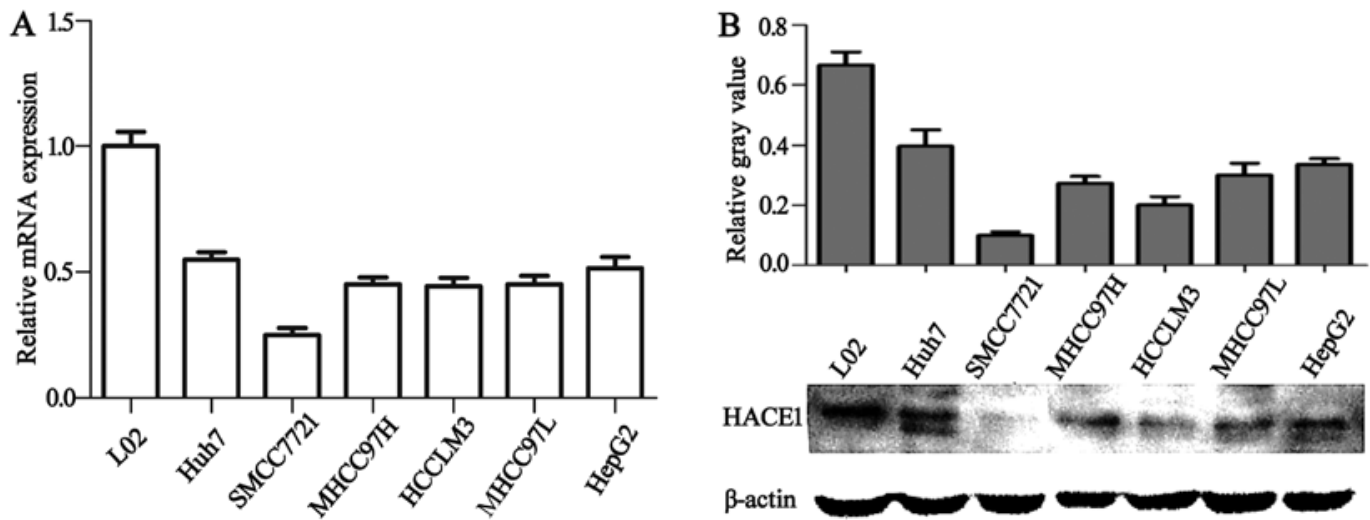

Figure 2. HACE1 expression in HCC cell lines and a normal liver cell line at both the mRNA and protein levels. (A) HACE1 mRNA expression levels in HCC cell lines and the normal liver cell line L02 by qRT-PCR analysis. (B) Western blot detection of HACE1 protein expression in HCC cell lines and the normal liver cell line L02. HACE1, HECT domain and ankyrin repeat containing E3 ubiquitin protein ligase 1; HCC, hepatocellular carcinoma.
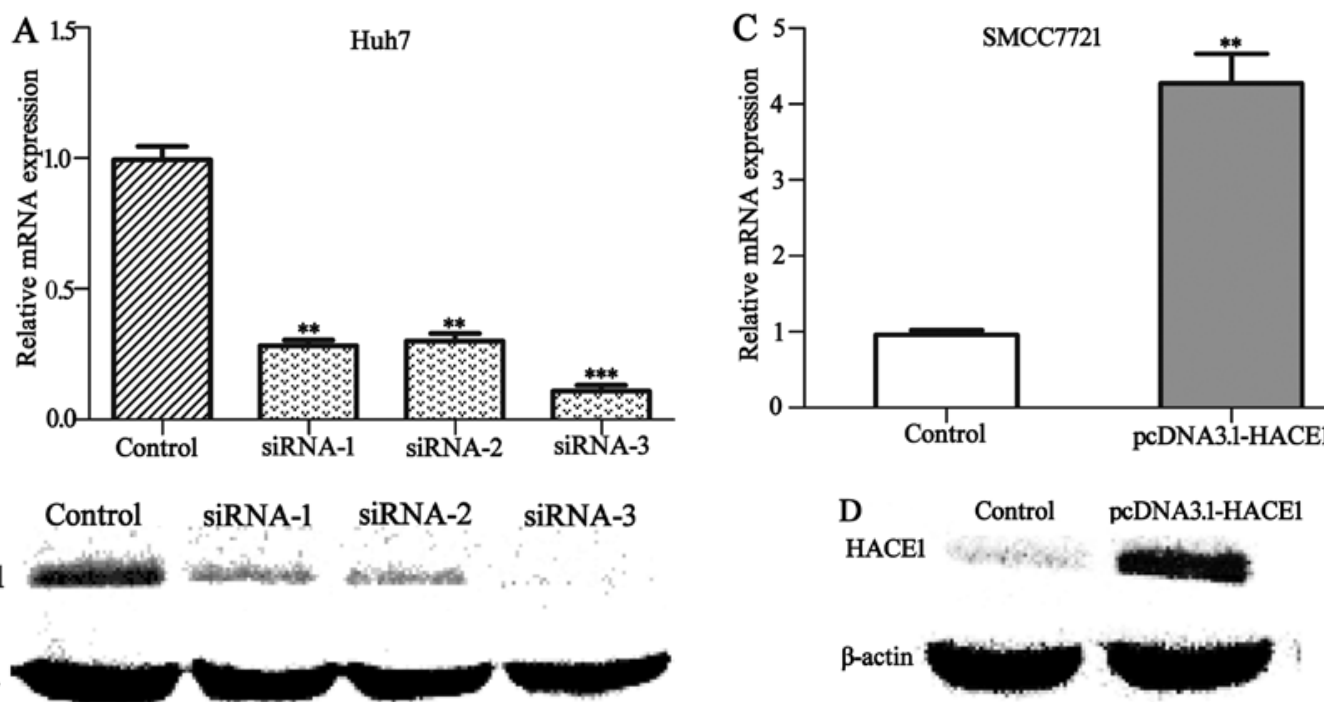

Figure 3. Confirmation of infection efficiency in the Huh7 and SMCC7721 cells. (A) Huh7 cells were infected with HACE1-siRNA or NC siRNA and the HACE1 mRNA levels were analyzed using qRT-PCR. (B) Western blot detection of the expression of HACE1 protein after infection. (C) SMCC7721 cells were infected with pcDNA3.1-HACE1 or mock plamid and HACE1 mRNA levels were analyzed using qRT-PCR. (D) Western blot detection of the expression of HACE1 protein after infection. Stably transfected Huh7 cells with HACE1-siRNA3 and SMCC7721 cells with pcDNA3.1-HACE1 were established successfully $\left({ }^{* *} \mathrm{P}<0.01,{ }^{* * *} \mathrm{P}<0.001\right)$. HACE1, HECT domain and ankyrin repeat containing E3 ubiquitin protein ligase 1.

HACE1 was markedly lower in the HCC tissues compared to that noted in the matched adjacent non-tumor tissues at both the protein and mRNA levels. The HACE1 protein and mRNA expression in $40 \mathrm{HCC}$ specimens and their adjacent non-tumor tissues were evaluated by western blot analysis and qRT-PCR. HACE1 mRNA expression level of HACE1 was lower in 32 of the $40 \mathrm{HCC}$ samples $(80 \%)$ than in the adjacent non-tumor tissues $(\mathrm{P}<0.01$; Fig. $1 \mathrm{~A})$. The protein expression of HACE1 was decreased in 30 of the 40 HCC samples (75\%), compared with the matched adjacent non-tumor tissues $(\mathrm{P}<0.001$; Fig. 1B and $\mathrm{C})$.

Expression of HACE1 protein and $\mathrm{mRNA}$ in HCC cell lines. We assessed the HACE1 gene expression in HCC cell lines by western blot analysis and qRT-PCR. Western blot analysis and qRT-PCR determined that HACE1 mRNA (Fig. 2A) and protein expression (Fig. 2B) were significantly decreased in the $6 \mathrm{HCC}$ cell lines compared with the expression levels in the normal liver cell line L02. As shown in Fig. 2, the highest expression of the HACE1 gene was detected in Huh7 cells, and the lowest was detected in the SMCC7721 cells among all HCC cell lines. Therefore, to verify the role of HACE1 in the HCC cell lines, we upregulated HACE1 by transfection of pcDNA3.1-HACE1 into the SMCC7721 cells and Huh7 cells were transfected with siRNA targeting HACE1 for downregulation.

Knockdown and overexpression efficiency as determined by western blot analysis and qRT-PCR. To explore the role of HACE1, we knocked down the HACE1 gene in the human HCC Huh7 cell line and overexpressed HACE1 in the HCC SMCC7721 cell line, respectively. As shown in Fig. 3A and B, by day 2 post infection, HACE1 protein and mRNA levels of HACE1-siRNA-infected Huh7 cells were significantly 

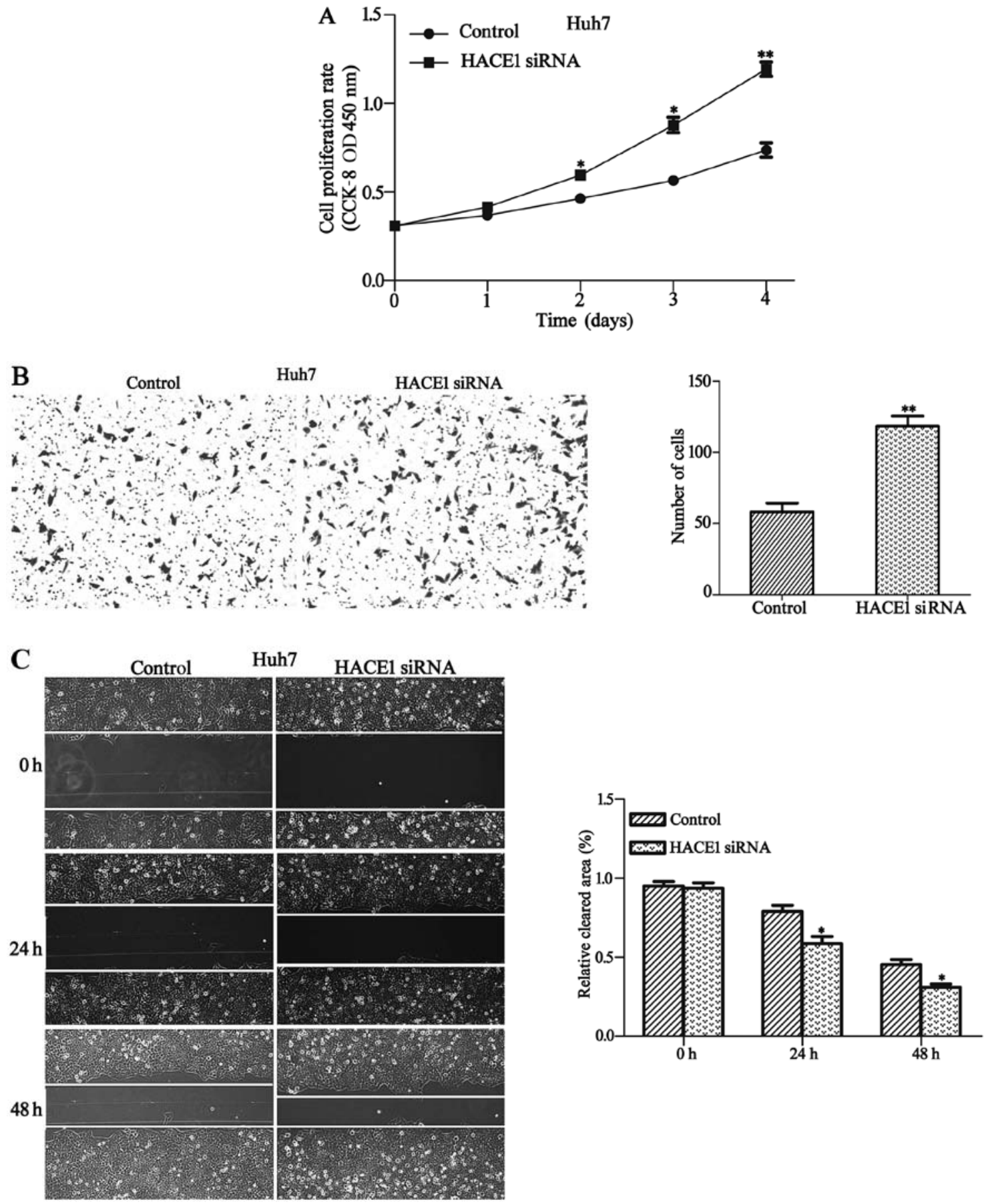

Figure 4. Effects of the knockdown of HACE1 on cell proliferation and migration in the Huh7 cell line. (A) The CCK-8 assay was performed following HACE1 siRNA3 infection, and cell proliferation of Huh7 cells was measured. (B) The migration of Huh7 cells was assessed by Transwell assay, (magnification, x100) and the number of migratory cells of six random fields was counted. (C) Wound healing assays of Huh7 cells. HACE1 knockdown had a measurable inhibitory effect on cell migration. Magnification, $\mathrm{x} 100$. Knockdown of HACE1 promoted the proliferation and migration of Huh7 cells ( $\mathrm{P}<0.05$, $\left.{ }^{* *} \mathrm{P}<0.01\right)$. The histograms depict the quantification of triplicate results with mean \pm SD. HACE1, HECT domain and ankyrin repeat containing E3 ubiquitin protein ligase 1.

lower compared to levels in the cultures infected with the NC siRNA by western blot analysis and qRT-PCR. The highest knockdown efficiency of HACE1 siRNA was siRNA-3 and thus siRNA-3 was used in the subsequent experiment $(\mathrm{P}<0.001)$. Similarly, HACE1 mRNA and protein levels of pcDNA3.1-HACE1-infected SMCC7721 cell line were significantly higher compared to levels in the cultures infected with the mock plamid as a control $(\mathrm{P}<0.01$; Fig. $3 \mathrm{C}$ and $\mathrm{D})$.
Effect of the knockdown and overexpression of HACE1 on cellular proliferation and migration in the Huh7 and SMCC7721 cell lines. To evaluate the role of HACE1 in HCC proliferation and migration, two different types of cells were examined by CCK-8, Transwell and wound healing assays. The results indicated that downregulation of HACE1 expression significantly inhibited the proliferation and migration of the Huh7 cells ( $\mathrm{P}<0.05$; Fig. 4A-C), and upregulation of HACE1 

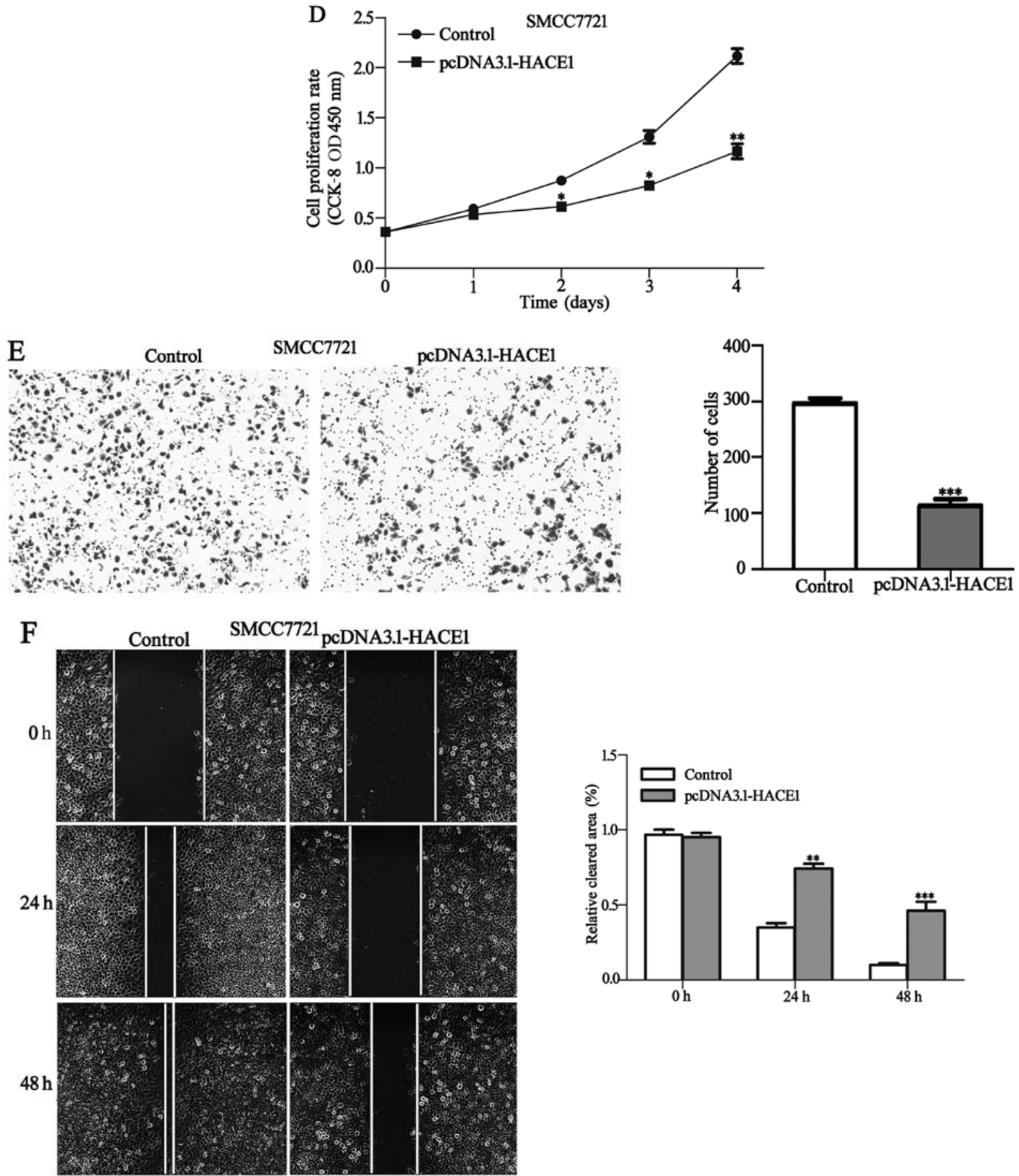

Figure 4. Continued. Effect of the overexpression of HACE1 on cell proliferation and migration in the SMCC7721 cell line. (D) The CCK-8 assay was performed following pcDNA3.1-HACE1 infection, and cell proliferation of the SMCC7721 cells was measured. (E) The migration of SMCC7721 cells was assessed by Transwell assay and the number of migratory cells for six random fields was counted. (F) Wound healing assays. HACE1 overexpression had a measurable promoting effect on the cell migration of the SMCC7721 cells. Magnification, x100. Overexpression of HACE1 inhibited the proliferation and migration of SMCC7721 cells $\left({ }^{* *} \mathrm{P}<0.01,{ }^{* * *} \mathrm{P}<0.001\right)$. The histograms depict the quantification of triplicate results with mean \pm SD. HACE1, HECT domain and ankyrin repeat containing E3 ubiquitin protein ligase 1.

expression obviously promoted the proliferation and migration of the SMCC7721 cells ( $\mathrm{P}<0.05$; Fig. 4D-F).

Correlation between HACE1 expression and clinicopathological characteristics. We explored the correlation of HACE1 expression and the clinicopathological characteristics of the 102 HCC patients based on IHC analysis (Fig. 5) and the results are summarized in Table I. From the results, we found that 75 samples (73.52\%) had low HACE1 expression, and 27 samples $(26.48 \%)$ had high HACE1 expression. According to the level of HACE1 expression in the tumor tissues, we demonstrated that a low level of HACE1 expression was significantly associated with serum AFP level $(\mathrm{P}<0.001)$, tumor differentiation $(\mathrm{P}<0.05)$ and vascular invasion $(\mathrm{P}<0.05)$. Overall survival was significantly decreased in the patients with low HACE1 expression compared to patients with high 

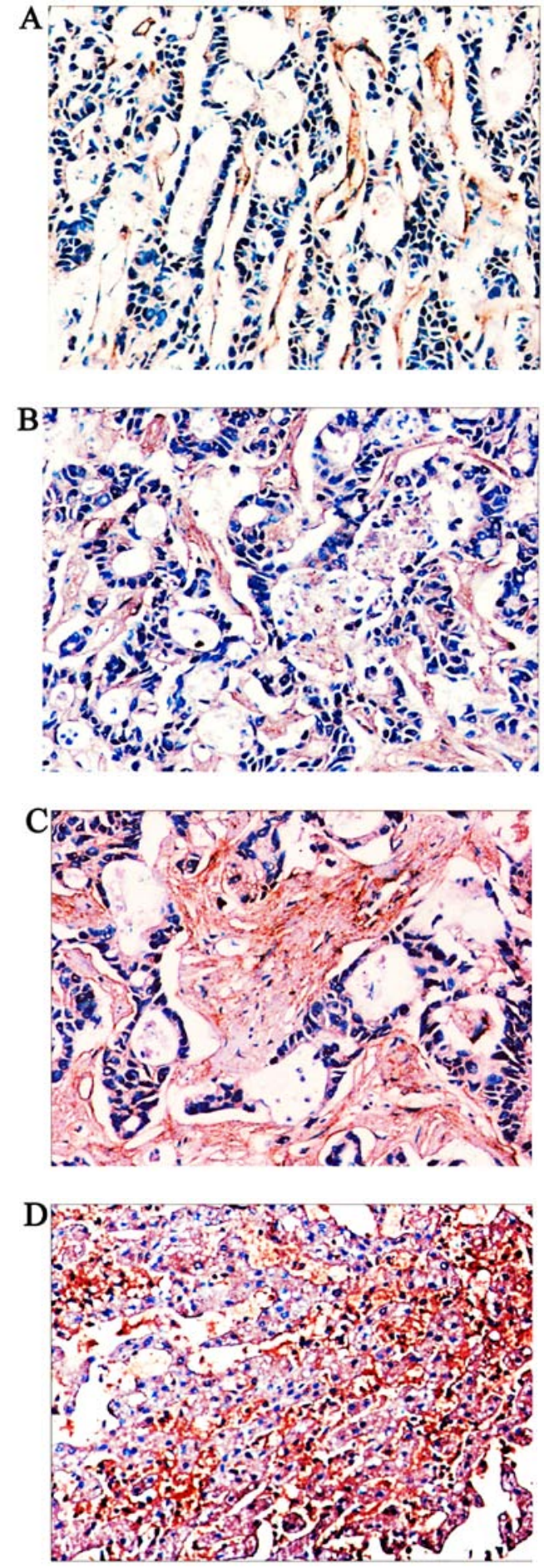

Figure 5. IHC analysis of HACE1 expression in HCC tissues and adjacent non-tumor tissues. (A) Low expression with weak staining in tumor tissue. (B) General expression with moderate staining in tumor tissue. (C) High expression with strong staining in tumor tissue. (D) High expression of HACE1 in adjacent non-tumor tissue. Magnification, x200. IHC, immunohistochemistry; HACE1, HECT domain and ankyrin repeat containing E3 ubiquitin protein ligase 1 .

HACE1 expression (Fig. 6). In addition, univariate and multivariate Cox regression analyses demonstrated that AFP level, tumor differentiation, Child-Pugh and HACE1 level were significantly associated with overall survival and were significant prognostic factors for HCC patients (Table II).
Table I. Correlation between HACE1 expression and clinicopathological characteristics of the HCC cases $(n=102)$.

\begin{tabular}{|c|c|c|c|c|}
\hline \multirow{2}{*}{$\begin{array}{l}\text { Clinicopathological } \\
\text { indices }\end{array}$} & \multicolumn{2}{|c|}{ HACE1 } & \multirow[b]{2}{*}{$\chi^{2}$} & \multirow[b]{2}{*}{ P-value } \\
\hline & Low & High & & \\
\hline \multicolumn{5}{|l|}{ Age (years) } \\
\hline$\leq 50$ & 38 & 12 & 0.308 & 0.580 \\
\hline$>50$ & 37 & 15 & & \\
\hline \multicolumn{5}{|l|}{ Gender } \\
\hline Female & 15 & 5 & 0.028 & 0.868 \\
\hline Male & 60 & 22 & & \\
\hline \multicolumn{5}{|l|}{$\mathrm{AFP}(\mathrm{ng} / \mathrm{ml})$} \\
\hline$\leq 20$ & 27 & 20 & 11.583 & $<0.001^{\mathrm{a}}$ \\
\hline$>20$ & 48 & 7 & & \\
\hline \multicolumn{5}{|l|}{$\operatorname{ALT}(\mathrm{U} / \mathrm{l})$} \\
\hline$\leq 75$ & 31 & 14 & 0.891 & 0.345 \\
\hline$>75$ & 44 & 13 & & \\
\hline \multicolumn{5}{|l|}{ Tumor size $(\mathrm{cm})$} \\
\hline$\leq 5$ & 46 & 16 & 0.036 & 0.850 \\
\hline$>5$ & 29 & 11 & & \\
\hline \multicolumn{5}{|l|}{ Tumor number } \\
\hline 1 & 56 & 20 & 0.004 & 0.952 \\
\hline$>1$ & 19 & 7 & & \\
\hline \multicolumn{5}{|l|}{ Vascular invasion } \\
\hline No & 41 & 21 & 4.449 & $0.035^{\mathrm{a}}$ \\
\hline Yes & 34 & 6 & & \\
\hline \multicolumn{5}{|l|}{ HBsAg } \\
\hline Positive & 59 & 17 & 2.578 & 0.108 \\
\hline Negative & 16 & 10 & & \\
\hline \multicolumn{5}{|l|}{ Tumor differentiation } \\
\hline $\mathrm{I} / \mathrm{II}$ & 42 & 22 & 5.515 & $0.019^{\mathrm{a}}$ \\
\hline III/IV & 33 & 5 & & \\
\hline \multicolumn{5}{|l|}{ Child-Pugh score } \\
\hline A & 27 & 14 & 2.075 & 0.150 \\
\hline B & 48 & 13 & & \\
\hline \multicolumn{5}{|l|}{ Liver cirrhosis } \\
\hline Positive & 60 & 17 & 3.115 & 0.078 \\
\hline Negative & 15 & 10 & & \\
\hline
\end{tabular}

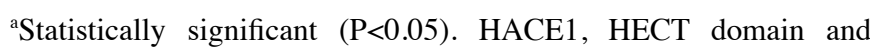
ankyrin repeat containing E3 ubiquitin protein ligase 1; HCC, hepatocellular carcinoma.

\section{Discussion}

Genomic losses or low expression in relation to human chromosome $6 \mathrm{q} 21$ have been described for a wide spectrum of tumor types including lung or breast cancer, as well as ovarian and gastric cancers $(6,17,18)$, suggesting that this region encompasses one or more major tumor-suppressor genes. The HACE1 gene encodes a $103-\mathrm{kDa}$ protein containing six $\mathrm{N}$-terminal ankyrin repeats connected via a linker region to a C-terminal HECT domain (19). The 
Table II. Univariate and multivariate analyses of the overall survival of 102 patients with HCC.

\begin{tabular}{|c|c|c|c|c|c|c|}
\hline \multirow[b]{2}{*}{ Variables } & \multirow[b]{2}{*}{ HR } & \multicolumn{2}{|c|}{ Univariate analysis } & \multirow[b]{2}{*}{ HR } & \multicolumn{2}{|c|}{ Multivariate analysis } \\
\hline & & $95 \% \mathrm{CI}$ & P-value & & $95 \% \mathrm{CI}$ & P-value \\
\hline Age (years) & 0.976 & $0.542-1.572$ & 0.784 & & & \\
\hline Gender & 0.624 & $0.338-1.334$ & 0.337 & & & \\
\hline $\operatorname{AFP}(\mathrm{ng} / \mathrm{ml})$ & 3.028 & $1.760-6.075$ & $<0.001^{\mathrm{a}}$ & 3.432 & $1.831-6.433$ & $<0.001^{\mathrm{a}}$ \\
\hline $\operatorname{ALT}(\mathrm{U} / \mathrm{l})$ & 0.951 & $0.531-1.543$ & 0.762 & & & \\
\hline Tumor size $(\mathrm{cm})$ & 1.295 & $0.881-2.018$ & 0.575 & & & \\
\hline Tumor number & 1.058 & $0.610-1.823$ & 0.658 & & & \\
\hline Vascular invasion & 1.032 & $0.595-1.818$ & 0.779 & & & \\
\hline HBsAg & 1.135 & $0.635-1.943$ & 0.643 & & & \\
\hline Tumor differentiation & 2.126 & $1.350-3.586$ & $0.011^{\mathrm{a}}$ & 1.892 & $1.214-2.721$ & $0.039^{\mathrm{a}}$ \\
\hline Child-Pugh score & 2.051 & $1.319-2.974$ & $0.013^{\mathrm{a}}$ & 1.987 & $1.285-3.768$ & $0.027^{\mathrm{a}}$ \\
\hline Liver cirrhosis & 1.259 & $0.796-1.986$ & 0.581 & & & \\
\hline HACE1 level & 2.432 & $1.486-4.571$ & $0.009^{\mathrm{a}}$ & 2.174 & $1.385-4.090$ & $0.013^{\mathrm{a}}$ \\
\hline
\end{tabular}

aStatistically significant $(\mathrm{P}<0.05)$. HCC, hepatocellular carcinoma.

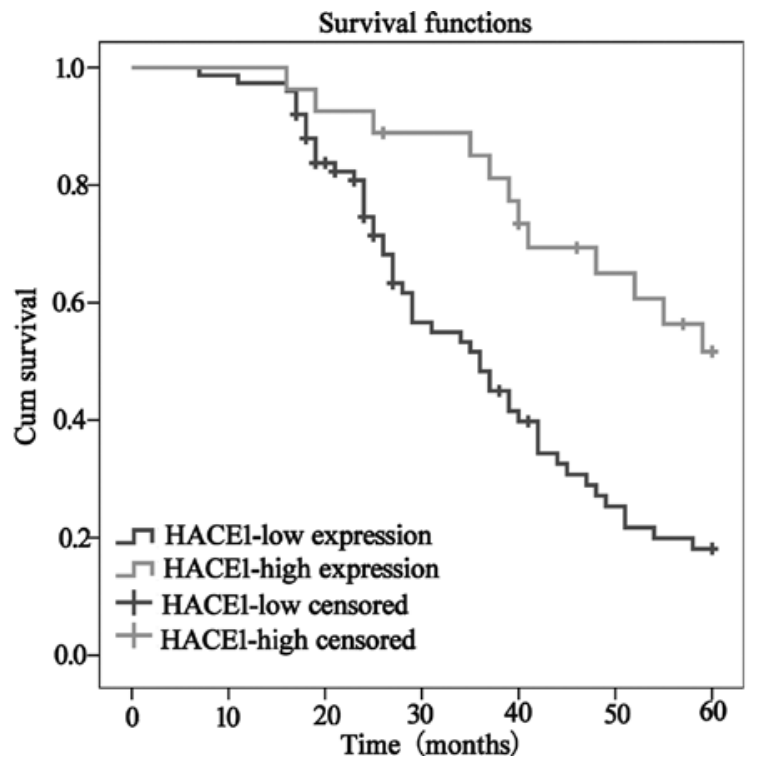

Figure 6. Kaplan-Meier analysis of overall survival in HCC patients with low or high HACE1 expression was evaluated using log-rank test $(\mathrm{P}<0.05)$. HACE1, HECT domain and ankyrin repeat containing E3 ubiquitin protein ligase 1; HCC, hepatocellular carcinoma.

expression of the 6q21 HACE1 gene is downregulated in multiple human tumor types involving Wilms' tumor, breast cancer and lung carcinoma (20-23). Findings of previous studies have demonstrated that HACE1 lower expression or deletion, as caused by HACE1 ubiquitination or methylation, is related to the occurrence and invasion in different types of carcinomas $(10,24,25)$. It was revealed that HACE1 is a new type of candidate anti-oncogene and perhaps a therapeutic target for several types of human cancers. However, to the best of our knowledge, the expression of HACE1 and its possible role in the anticancer effects on hepatocellular carcinoma have not been discussed to date.

In the present study, we firstly found that the HACE1 expression level was downregulated at both the mRNA and protein levels in HCC tissues and HCC cell lines when compared with these levels in adjacent non-tumor tissues and a normal cell line L02 as analyzed by qRT-PCR and western blot analysis. Thus, we assumed that HACE1 plays an important role in tumor proliferation and migration. To explore this issue, CCK-8, Transwell and wound healing assays were performed. The result demonstrated that overexpression of HACE1 by transfection with pcDNA3.1-HACE1 in SMCC7721 cells led to lower proliferation and migration ability compared to the control group. In addition siRNA-mediated HACE1 knockdown in Huh7 cells significantly accelerated cell proliferation and migration. The downregulation of HACE1 expression correlated with the serum AFP level, tumor differentiation and vascular invasion in HCC patients. Survival analyses exhibited that patients with a low HACE1 expression showed poorer overall survival than those with a high HACE1 expression, and the status of HACE1 expression was an independent prognostic factor.

In conclusion, we provided strong evidence that HACE1 was significantly downregulated in HCC cell lines and HCC tissues. Moreover, the expression of HACE1 in HCC tissues was associated with the development, progression and metastasis of $\mathrm{HCC}$ or prognosis of the patients by regulating the proliferation and migration of HCC cells. IHC analysis suggested that the expression of HACE1 in HCC tissues was associated with serum AFP level, tumor differentiation and vascular invasion. Survival analyses demonstrated that patients with low HACE1 expression exhibited poorer overall survival than those with high HACE1 expression, and the level of HACE1 expression was an independent prognostic factor.

In addition, in vitro studies showed that overexpression of HACE1 decreased the proliferation and migration ability in the human HCC cell line SMCC7721. On the contrary, HACE1 
knockdown in human cell line Huh7 facilitated the proliferation and migration ability. Yet, further exploration is required to elucidate the signaling pathways and molecular mechanism of HACE1 in HCC, which may be useful in acquiring better understanding of the molecular pathogenesis of these tumors. Taken together, the results suggest that, as a tumor-suppressor, HACE1 can be used to develop more effective targeted therapeutic strategies and may be a potential therapeutic target for HCC treatment.

\section{Acknowledgements}

This study was supported by grants from the National Natural Science Foundation of China (grant nos. 31270543 and 81101818) and Fundamental Research Funds for the Central Universities (lzujbky-2013-k21).

\section{References}

1. Torre LA, Bray F, Siegel RL, Ferlay J, Lortet-Tieulent J and Jemal A: Global cancer statistics, 2012. CA Cancer J Clin 65: 87-108, 2015.

2. Waller LP, Deshpande V and Pyrsopoulos N: Hepatocellular carcinoma: A comprehensive review. World J Hepatol 7: 2648-2663, 2015.

3. Liccioni A, Reig M and Bruix J: Treatment of hepatocellular carcinoma. Dig Dis 32: 554-563, 2014.

4. Waghray A, Murali AR and Menon KN: Hepatocellular carcinoma: From diagnosis to treatment. World J Hepatol 7: 1020-1029, 2015.

5. Anglesio MS, Evdokimova V, Melnyk N, Zhang L, Fernandez CV, Grundy PE, Leach S, Marra MA, Brooks-Wilson AR, Penninger J, et al: Differential expression of a novel ankyrin containing E3 ubiquitin-protein ligase, Hace1, in sporadic Wilms' tumor versus normal kidney. Hum Mol Genet 13: 2061-2074, 2004.

6. Zhang L, Anglesio MS, O'Sullivan M, Zhang F, Yang G, Sarao R, Mai PN, Cronin S, Hara H, Melnyk N, et al: The E3 ligase HACE1 is a critical chromosome 6q21 tumor suppressor involved in multiple cancers. Nat Med 13: 1060-1069, 2007.

7. Qu Y, Dang S and Hou P: Gene methylation in gastric cancer. Clin Chim Acta 424: 53-65, 2013.

8. Sako N, Dessirier V, Bagot M, Bensussan A and Schmitt C: HACE1, a potential tumor suppressor gene on $6 \mathrm{q} 21$, is not involved in extranodal natural killer/T-cell lymphoma pathophysiology. Am J Pathol 184: 2899-2907, 2014.

9. Liu Z, Chen P, Gao H, Gu Y, Yang J, Peng H, Xu X, Wang H, Yang M, Liu X, et al: Ubiquitylation of autophagy receptor Optineurin by HACE1 activates selective autophagy for tumor suppression. Cancer Cell 26: 106-120, 2014.

10. Mettouchi A and Lemichez E: Ubiquitylation of active Racl by the E3 ubiquitin-ligase HACE1. Small GTPases 3: 102-106, 2012.
11. Zhang L, Chen X, Sharma P, Moon M, Sheftel AD, Dawood F, Nghiem MP, Wu J,Li RK, Gramolini AO, et al: HACE1-dependent protein degradation provides cardiac protection in response to haemodynamic stress. Nat Commun 5: 3430, 2014.

12. Deng $S$ and Huang C: E3 ubiquitin ligases in regulating stress fiber, lamellipodium, and focal adhesion dynamics. Cell Adhes Migr 8: 49-54, 2014.

13. Castillo-Lluva S, Tan CT, Daugaard M, Sorensen PH and Malliri A: The tumour suppressor HACE1 controls cell migration by regulating Racl degradation. Oncogene 32: 1735-1742, 2013.

14. Goka ET and Lippman ME: Loss of the E3 ubiquitin ligase HACE1 results in enhanced Rac1 signaling contributing to breast cancer progression. Oncogene 34: 5395-5405, 2015.

15. Huang Y, de Reyniès A, de Leval L, Ghazi B, Martin-Garcia N, Travert M, Bosq J, Brière J, Petit B, Thomas E, et al: Gene expression profiling identifies emerging oncogenic pathways operating in extranodal NK/T-cell lymphoma, nasal type. Blood 115: 1226-1237, 2010.

16. Hibi K, Sakata M, Sakuraba K, Shirahata A, Goto T, Mizukami H, Saito M, Ishibashi K, Kigawa G, Nemoto H, et al: Aberrant methylation of the HACE1 gene is frequently detected in advanced colorectal cancer. Anticancer Res 28 (3A): 1581-1584, 2008.

17. Hudson C, Schwanke C, Johnson JP, Elias AF, Phillips S, Schwalbe T, Tunby M and Xu D: Confirmation of 6q21-6q22.1 deletion in acro-cardio-facial syndrome and further delineation of this contiguous gene deletion syndrome. Am J Med Genet A 164A: 2109-2113, 2014.

18. Wang XC, Zhang JQ, Shen YQ, Miao FQ and Xie W: Loss of heterozygosity at $6 \mathrm{p} 21.3$ underlying HLA class I downregulation in gastric cancer. J Exp Clin Cancer Res 25: 115-119, 2006.

19. Scheffner M and Kumar S: Mammalian HECT ubiquitin-protein ligases: Biological and pathophysiological aspects. Biochim Biophys Acta 1843: 61-74, 2014.

20. Stewénius Y, Jin Y, Ora I, Panagopoulos I, Möller E, Mertens F, Sandstedt B, Alumets J, Akerman M, Merks JH, et al: High-resolution molecular cytogenetic analysis of Wilms tumors highlights diagnostic difficulties among small round cell kidney tumors. Genes Chromosomes Cancer 47: 845-852, 2008.

21. Capasso M,DiskinS,CimminoF,Acierno G, Totaro F,Petrosino G, Pezone L, Diamond M, McDaniel L, Hakonarson $\mathrm{H}$, et al: Common genetic variants in NEFL influence gene expression and neuroblastoma risk. Cancer Res 74: 6913-6924, 2014.

22. Capasso M, Diskin SJ, Totaro F, Longo L, De Mariano M, Russo R, Cimmino F, Hakonarson H, Tonini GP, Devoto M, et al: Replication of GWAS-identified neuroblastoma risk loci strengthens the role of BARD1 and affirms the cumulative effect of genetic variations on disease susceptibility. Carcinogenesis 34: 605-611, 2013

23. Küçük C, Hu X, Iqbal J, Gaulard P, Klinkebiel D, Cornish A, Dave BJ and Chan WC: HACE1 is a tumor suppressor gene candidate in natural killer cell neoplasms. Am J Pathol 182: 49-55, 2013.

24. Gacon G, Mettouchi A and Lemichez E: The tumor suppressor HACE1 targets Rac1 to ubiquitin-mediated proteasomal degradatio]. Med Sci (Paris) 28: 39-41, 2012 (In French).

25. Lachance V, Degrandmaison J, Marois S, Robitaille M, Génier S, Nadeau S, Angers $S$ and Parent JL: Ubiquitylation and activation of a Rab GTPase is promoted by a $\beta_{2}$ AR-HACE1 complex. J Cell Sci 127: 111-123, 2014. 